\title{
Nanotechnological Enhancement of Infrared Detectors by Plasmon Resonance in Transparent Conductive Oxide Nanoparticles
}

\author{
Zoran Jakšić1,* - Momčilo Milinović2 - Danijela Randjelović1 \\ ${ }^{1}$ Institute of Chemistry, Technology and Metallurgy, University of Belgrade, Serbia \\ 2 Faculty of Mechanical Engineering, University of Belgrade, Serbia
}

\begin{abstract}
We investigated the use of plasmonic nanotechnology to enhance the performance of semiconductor infrared detectors. An increase of quantum efficiency, responsivity and specific detectivity is obtained by applying transparent conductive oxide (TCO) nanoparticles onto the surface of a photodetector. To this purpose we considered uncooled mercury cadmium telluride (HgCdTe) photoconductive detectors fabricated by isothermal vapor phase epitaxy, but the same procedure can be applied to cryogenically cooled devices, including those of photovoltaic type. The main mechanism of enhancement is light concentration ensured through localized plasmon resonance at the TCO nanoparticles and through enhanced scattering, while the desired wavelength range is reached by a further redshifting through the adjustment of nanoparticle properties. The improvement can be implemented during the final stages of production of the existing photovoltaic and photoconductive detectors. The method is applicable to various practical applications, including updating of high-precision guided ammunition.
\end{abstract}

Keywords: nanotechnology, homing head, infrared detectors, plasmonic enhancement, transparent metal oxides, nanoparticles

\section{O INTRODUCTION}

The possibility of detecting and recognizing objects under low light conditions [1] has applications in many fields, from personal and instrumental surveillance to homing devices utilizing heat seeking guidance. Advanced technological solutions for missile guidance provided in the past have later spread not only to missiles but also to classic projectiles like mortar shells, gun shells, etc., thus forming the basis for "smart ammunition".

Single-shot lethality is becoming the most important quality requirement for both missiles and guided projectiles. New mortar shells technology [2] and [3], developed in the form of missiles with homing heads, usually depends either on laser illumination of the target for its guidance or an autonomous selfguided heat-seeking mode, as in the case of terminal infrared homing. When using terminal guidance for projectiles the main goal is to develop the recognition of signals reflected from illuminated targets, to hit and kill, to a level of a reliable technical characteristic in the required chain.

This is especially important for fighting any type of combat armored targets in motion, which requires target tracking by laser designator during the projectile terminal approaching phase.

The sensor on the homing head of a guided projectile requires highly sensitive optical detection to recognize reflected laser signals from the target illuminated by a designator and to provide an appropriate signal for the flight control correction of the missile or projectile. The control of the missile or the projectile at the last phase of ballistic trajectory at distances less than $1000 \mathrm{~m}$ requires fast influence on the changes of the missile's axes or velocity vector direction toward the mobile target. For this type of control [3] the element with the largest influence on the missile shooting performances is the type of detector in the homing head, which has to be sufficiently sensitive to measure the flux and the direction of the light impulse reflected from the illuminated target. The whole process is extremely rapid and the reaction of the homing head and the missile control mechanism directly influence target shooting errors. Since artillery projectiles [3] must be low-cost, compact, rugged and simple, this means that the implementation of the detection function must be performed without additional complex equipment for improving the sensitivity of light flux detection in the homing head sensor. Self-guidance homing heads operate without illumination sources and use infrared (IR) signature of targets to recognize the guidance LOS (line of sight) direction. This means that high response speed and high sensor sensitivity under poor lighting condition and at infrared wavelengths where, typically, the influence of noise and interference is much larger than in visible and further increases with wavelength, must be ensured.

It is well known that semiconductor infrared detectors typically ensure the highest response speed of all detector types [1] and [4]. They do not depend on thermal diffusion at all, contrary to the thermal detectors, but are excited directly by infrared radiation from the target through interband transitions of charge carriers. Such direct conversion from light to electric signal ensures rapid reaction and because of that such detectors are the devices of choice in military systems. 
Another issue is how to improve the sensitivity (responsivity) and specific detectivity of infrared detector without simultaneously increasing the system complexity and cost. To this purpose one has to ensure a maximization of light flux within the active area of the detector. This belongs to the field of light management, which is one of the most significant issues with photodetectors generally, regardless of the wavelength range. It is of especial importance for the new homing heads utilizing either laser-illuminated or self-guided missiles and projectiles technology [2] and [3].

The aim of this paper is to present a possible nanotechnological solution for highly sensitive and low-cost infrared semiconductor detectors applicable for various purposes, including updating high-precision guided ammunition. To this purpose, mercury cadmium telluride uncooled photoconductive detectors fabricated by vapor phase epitaxy were considered. Aluminum-doped zinc oxide and tin oxide nanoparticles fabricated by nonaqueous procedure were considered for the improvement of detector samples. The original research results also include the assessment of the optimization wavelength range through redshifting of nanoparticle optical properties.

\section{REQUIREMENTS AND CONSTRAINTS FOR INFRARED DETECTORS}

Active infrared systems, including those for homing heads, utilize as their main principle the detection of infrared radiation, usually at wavelengths belonging to the (3 to 5$) \mu \mathrm{m}$ or (8 to 14) $\mu \mathrm{m}$ atmospheric windows. The infrared photodetectors typically used for these ranges are either silicon thermal devices like bolometers, thermocouples, etc. (low-end and slower, but less expensive), or semiconductor detectors utilizing narrow-bandgap materials like indium antimonide or mercury cadmium telluride (much higher performance, a typical choice for military applications). Semiconductor devices offer much higher speeds, high specific detectivities with background-limited infrared photodetector (BLIP) operation and sharply defined operating wavelength ranges. Our present analysis is dedicated to this type of detectors.

Mercury cadmium telluride $\left(\mathrm{Hg}_{1-\mathrm{x}} \mathrm{Cd}_{\mathrm{x}} \mathrm{Te}, \mathrm{MCT}\right)$ has been the infrared semiconductor material of choice for the last few decades [4]. The possibility to tailor its cadmium molar fraction $x$ during fabrication and thus to design a desired cutoff wavelength makes it useful for various detection ranges, from near to far infrared. The most sensitive $\mathrm{Hg}_{1-\mathrm{x}} \mathrm{Cd}_{\mathrm{x}} \mathrm{Te}$ infrared detectors are the cryogenically cooled ones [1], typically by liquid nitrogen or possibly by multi-stage thermoelectric coolers. However, there is also a class of $\mathrm{Hg}_{1-\mathrm{x}} \mathrm{Cd}_{\mathrm{x}} \mathrm{Te}$ infrared photodetectors that operate at room temperature, furnishing specific detectivities in excess of $10^{8} \mathrm{cmHz}^{1 / 2} / \mathrm{W}$ [5]. Fabrication of these $\mathrm{Hg}_{1-\mathrm{x}} \mathrm{Cd}_{\mathrm{x}} \mathrm{Te}$ infrared photodetectors using isothermal vapor phase epitaxy (ISOVPE) is presented in [6] and by liquid phase epitaxy (LPE) in [7].

It is well known that the performance of an infrared detector is described by its sensitivity and specific detectivity [1]. Sensitivity (also denoted as responsivity) is defined as a ratio of the incident infrared flux and the useful signal, which may be either voltage or current. The specific detectivity is further defined as the product of sensitivity and the squared active area divided by detector noise equivalent power. All of the exploitation demands quoted in the Introduction practically require the maximization of the specific detectivity in order to ensure the photodetector operation under weak illumination condition. On the other hand, this signifies there is a necessity to trap the maximum amount of the useful signal within the active region of the photodetector in order to optimize sensitivity (light management within the detector). This condition is especially important if the detector active areas are thin, as is usually the case with both ISOVPE and LPE epitaxial layers, but also for other devices from different materials and for other wavelength ranges, including e.g. ultrathin-film solar cells.

Various strategies have been developed to improve the light concentration within the photodetector, including the use of immersion lenses, antireflection coatings and structures, surface reliefs, highly reflective detector back sides, etc. [8].

Among the most recently proposed methods to improve the light flux within the photodetectors is the use of plasmon resonance [9]. Under certain conditions, an interface between dielectric and a material with negative relative dielectric permittivity (for instance good metals like silver or gold) will maintain electromagnetic waves confined to the interface and evanescent (i.e. exponentially decaying) in both directions from the interface surface. Such waves are termed surface plasmons polaritons (SPP). Plasmon waves may propagate along a planar guide, or be localized e.g. on the surface of nanoparticles (localized SPP). In each of these situations they ensure extremely large concentrations of electromagnetic field. Owing to this, they are very convenient for the enhancement of the operation of photodetectors generally, if the region of the concentrated field 
coincides with the active region of the photodetector. Obviously, this is especially useful for very thin detectors. Some methods for plasmonic enhancement that could be further modified for military grade detectors of reflected laser beam were originally proposed for photovoltaic solar cells [10] to [13].

The main problem with the use of plasmonic enhancement for infrared detector is that plasma resonance frequency at which the evanescent wave reaches its peak for metals is in the ultraviolet or visible part of the spectrum [14]. Thus, it is necessary to redshift the plasma frequency towards longer operating wavelengths. Several strategies were proposed to overcome this. One of them is to form complex patterns on detector surface which may serve as nanoaperture arrays [15] and [16] and which support the existence of the so-called designer ("spoof") plasmons [17]. In this way, the operating wavelength can be tailored to be proportional to the aperture dimensions and the spacing between them. Wavelengths 7 to $8 \mu \mathrm{m}$ were obtained in this manner [16]. Another redshifting strategy was to immerse metal nanoparticles into a higher refractive index material, which resulted in shifting the characteristics from the visible part of the spectrum to the wavelengths of up to $1.5 \mu \mathrm{m}$ [9]. Nanoparticles with sandwich structures were also utilized [16] where e.g. non-plasmonic material is covered by a thin shell of plasmonic material or there are several alternating layers of plasmonic and dielectric materials. Finally, one may use alternative low-loss plasmonic materials with intrinsically redshifted plasma frequency, like those described in [18].

In this work we consider possible novel strategies to further improve infrared detection in semiconductor detectors utilizing plasmonics. We consider the replacement of metal nanoparticles by transparent conductive oxides like tin oxide, indium oxide and zinc oxide. We also investigate the possibility to combine these materials with higher-index surrounding dielectric media and with additional patterning to obtain plasmonic scatterers enhancing the performance of thin-film infrared detectors.

\section{TECHNOLOGICAL MECHANISM OF LIGHT TRAPPING USING PLASMONIC NANOPARTICLES}

In this section, we briefly outline the possibility to enhance the quantum efficiency (the number of photocarriers generated by a single incident photon) and specific detectivity of a photodetector (regardless of its wavelength range) when there is only a micrometer-thin active area. To this purpose we consider a typical composition profile of an $\mathrm{Hg}_{1-\mathrm{x}} \mathrm{Cd}_{\mathrm{x}} \mathrm{Te}$ photodetector, as defined by its cadmium molar fraction $x$. An experimental dependence of $x$ on the distance from the detector surface is shown in Fig. 1a. The profile was experimentally determined from an ISOVPE-produced detector by IR transmission measurement because the cut-off wavelength of an MCT detector is defined by the lowest value of $x$, which is the one near the detector surface (left part of Fig. 1a). In several successive steps a submicrometerthin layer was removed from the detector surface utilizing chemical etching and for each thus obtained remaining structure its cut-off was again determined by IR transmission measurement, while the thickness of the remaining structure was measured utilizing a profilometer.
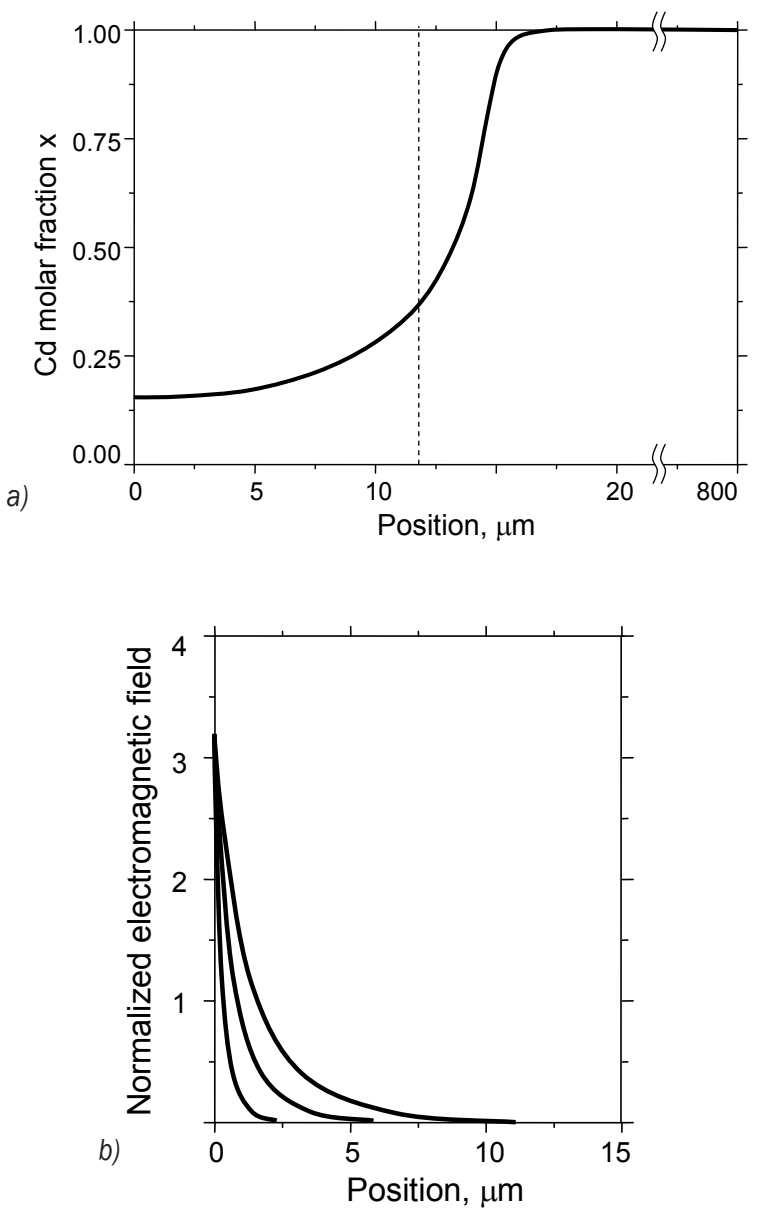

Fig. 1. a) measured $\mathrm{Cd}$ molar fraction profile of an MCT layer fabricated by open tube isothermal vapor phase epitaxy at a CdTe substrate; maximum detector response corresponds to the lowest Cd molar fraction, b) intensity of localized evanescent field near the detector surface in the case when a plasmonic layer is deposited on the surface; maximum detector response overlaps with maximum field enhancement 
Since the procedure of epitaxial growth allows the value of $x$ to be tuned in the whole range 0 to 1 , it is possible to easily tailor the detector response to a desired detector wavelength range and operating temperature. For instance, the optimum specific detectivity at room temperature and for a $10.6 \mu \mathrm{m}$ wavelength is obtained for a cadmium molar fraction $x=0.165$, while at $77 \mathrm{~K}$ and for the same wavelength the value should be $x=0.20$.

It can be seen that the thickness of the low- $x$ part is very small, its typical value being 5 to $15 \mu \mathrm{m}$. Thus, it is of interest to locate most of the incident optical signal within this part in order to maximize useful absorption. This is where plasmonic enhancement steps in. The plasmonic field is the largest at the surface and exponentially decreases towards the depth of semiconductor. The penetration depth depends on the thickness of the plasmonic material film at the surface and is larger for thinner plasmonic layers.

Such structures are very convenient since the photodetector for a given temperature and wavelength range can be customized by simply tailoring the cadmium molar fraction $x$. The optical field should be concentrated within the first several micrometers of their active area, and SPP fields suit that purpose well.

Fig. 2a shows the manner in which plasmonic nanoparticles can be utilized to enhance the field in the desired part. Each nanoparticle serves as a scatterer, and its plasmonic characteristics help direct most of the incident light into the active area. In addition to that, the above mentioned enhancement of the field around nanoparticles helps keep most of the light trapped within the near-surface part of the detector volume.

The localized electromagnetic field distribution around a nanoparticle is also shown in Fig. 2 for different situations. This is a qualitative presentation only and does not reflect the exact spatial distribution of the field. In all situations the external electromagnetic wave arrives from top. Fig. 2b shows the simplest case when the nanoparticle (black dot in the middle) is embedded in homogeneous medium. The distribution of the field (shaded grey circle) is symmetrical in forward and backward directions and is decreasing exponentially away from the nanoparticle (higher field is shown by lighter shading). Fig. 2c shows the field distribution when the nanoparticle is at an interface between media 1 and 2, where the refractive index of the bottom medium (2) is larger - the field is preferentially scattered towards the larger index medium. Fig. $2 d$ again shows the electromagnetic field distribution for a nanoparticle at an interface, but this time the refractive index of the top medium (1) is larger. Thus, the incident wave is always "folded" or "trapped" into a thin layer around the nanoparticle.

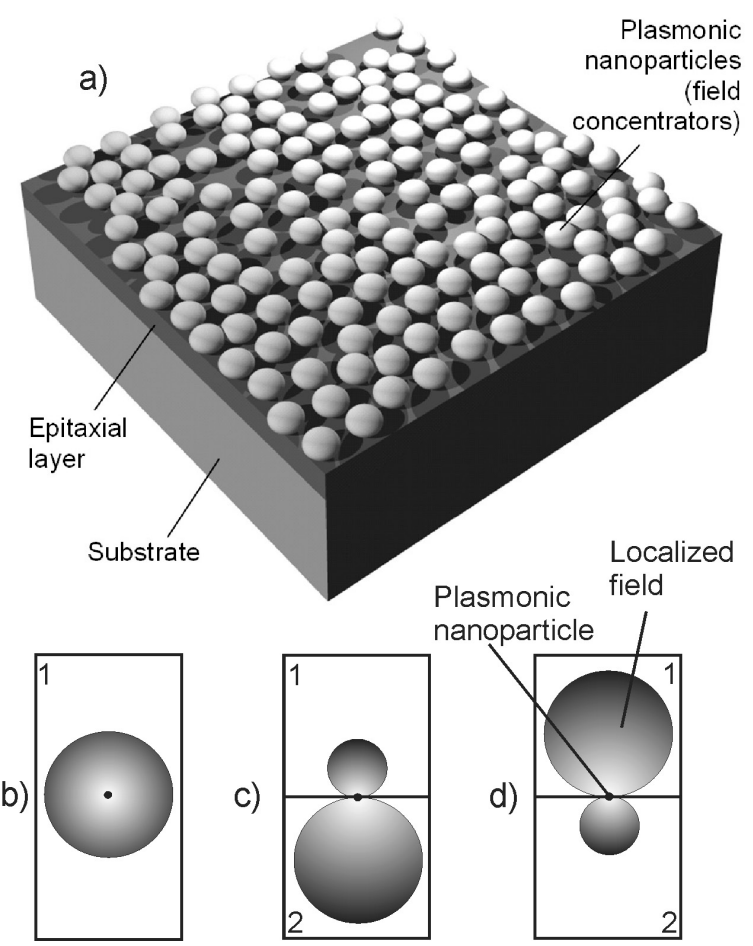

Fig. 2. Light trapping utilizing plasmonic nanoparticles; a) nanoparticles stochastically placed on the detector surface; b) field localization for a nanoparticle embedded in homogeneous medium (black dot in the centre: nanoparticle; gray circle: enhanced field, darker region corresponding to lower field); c) field localization for a nanoparticle at an interface between lower (1) and higher (2) refractive index medium; d) field localization if higher index medium is (1) and lower (2)
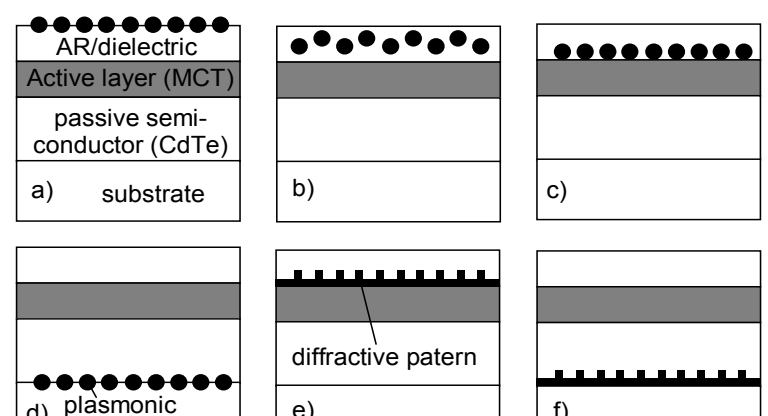

d) plasmonic
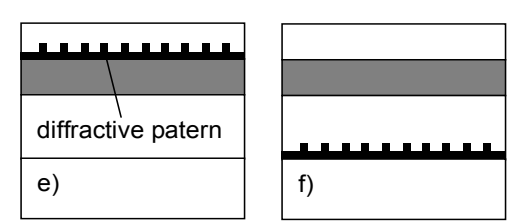

Fig. 3. Plasmonic structures for light concentration; a) nanoparticles on top of dielectric layer; b) nanoparticles embedded in dielectric; c) nanoparticles on top of active area (case shown in Fig. 1); d) nanoparticles on back side; e) diffractive pattern on top of active area; $f$ ) back-side diffractive pattern

Fig. 3 shows some possible geometries to achieve this. Nanoparticles may be placed on the surface 
dielectric layer (antireflection coating, AR), within that coating, or directly on the active surface (the case shown in Figs. 3a to c). In the cases Figs. 3a and c light trapping is represented by Fig. 2c, and in the case 3 by Fig. $2 b$. Nanoparticles may also be positioned on the detector back side, Fig. 3d. An alternative approach, depicted in Fig. $3 \mathrm{e}$ and $\mathrm{f}$ is the use of regular patterns which include diffractive gratings, but also subwavelength nanoaperture arrays with extraordinary optical transmission. Such regular patterns may be located at the detector surface (Fig. 3e), or at its back side (Fig. 3f). The trapping in the situations 3d, $\mathrm{e}$, and $\mathrm{f}$ is represented by Fig. $2 \mathrm{~d}$, since the detector active area has a higher value of refractive index.

\section{ENHANCING INFRARED DETECTOR PERFORMANCE USING TRANSPARENT CONDUCTIVE OXIDES}

Transparent conductive oxides (TCO) are plasmonic materials with their plasma frequency in infrared (compared to good metals where plasma frequency is in ultraviolet). Their relative dielectric permittivity is negative near plasma frequency, and its dispersion is well-described by electron resonance models of Drude or Lorentz [19] to [21]. The Drude model taking into account absorptive losses is:

$$
\varepsilon=\varepsilon_{\infty}-\frac{\omega_{p}^{2}}{\omega(\omega+i \Gamma)},
$$

where $\omega_{p}$ is the plasma frequency, $\Gamma$ denotes damping factor describing losses (i.e. defines the imaginary part of the complex dielectric permittivity), while $\varepsilon_{\infty}$ is the asymptotic relative dielectric permittivity.

The plasma frequency is determined by the properties of free carriers in material as:

$$
\omega_{p}^{2}=\frac{n e^{2}}{m^{*} \varepsilon_{0}},
$$

where $n$ is electron concentration, $e$ is the free electron charge $\left(1.6^{-10^{-19}} \mathrm{C}\right), \varepsilon_{0}$ is the free space (vacuum) permittivity $\left(8.854 \cdot 10^{-12} \mathrm{~F} / \mathrm{m}\right)$, and $m^{*}$ is the electron effective mass.

The damping factor can be calculated from the material scattering data as:

$$
\Gamma=\frac{e}{\mu m^{*}},
$$

where $\mu$ is mobility of free carriers in TCO.

The scattering cross-section of a particle is greatly enhanced by plasma resonance and may reach values up to 10 times larger than the geometrical cross-section. This means that a surface coverage of about $10 \%$ should be sufficient to capture practically $100 \%$ of the incident light and convert it into surface plasmons polaritons. The scattering cross-section for plasmonic nanoparticles at a wavelength $\lambda$ can be calculated utilizing the quasi-static approximation as [22]:

$$
C_{\text {scat }}=\frac{8}{3 \pi} \alpha^{2}\left[\frac{\pi}{\lambda}\right]^{4}
$$

where $\alpha$ is polarizability, with a functional form identical to that of the Clausius-Mossotti relation [23]:

$$
\alpha=3 V \frac{\frac{\varepsilon_{n p}}{\varepsilon_{d}}-1}{\frac{\varepsilon_{n p}}{\varepsilon_{d}}+2} .
$$

Here $\varepsilon_{n p}$ is the complex and wavelengthdispersive relative dielectric permittivity of the plasmonic nanoparticles, defined by Eq. (1), $\varepsilon_{d}$ is the permittivity of the surrounding dielectric medium and $V$ is the geometrical volume of the nanoparticle. The plasmon resonance and the maximum scattering crosssection are achieved at $\varepsilon_{n p}=-2 \cdot \varepsilon_{d}$. The absorption cross-section is determined as:

$$
C_{a b s}=\frac{2 \pi}{\lambda} \operatorname{Im}(\alpha) \text {. }
$$

It is important to note that losses within TCO nanoparticles are smaller than those in metals. At the same time, the plasmon resonance wavelength in such nanoparticles is shifted into the infrared part of the spectrum. The particular value of the resonant wavelength can be tailored by doping of TCO. Both facts are very important for the enhancement of the performance of the infrared semiconductor detectors

\section{REDSHIFTING STRATEGIES FOR TCO NANOPARTICLES}

In this section the properties of some possible photodetector enhancement configurations utilizing transparent conductive oxide nanoparticles or full oxide layers are assessed. A situation where plasmonic nanoparticles are deposited directly to the surface of the detector, i.e. the configuration shown in Fig. 2c is considered. An additional antireflection coating can be located over the plasmonic layer.

We analyzed two different TCO nanoparticle materials, tin oxide (TO) and aluminum-doped zinc oxide (AZO). The TO nanoparticles are fabricated 
utilizing a non-aqueous approach in autoclaves starting from a simple solution of $\mathrm{SnCl}_{4}$ in benzyl alcohol, while $\mathrm{AZO}$ is produced in pressurized reaction vessels in a microwave system. The nanoparticles size and morphology were characterized by scanning electron microscopy (SEM). Details on the fabrication and characterization procedures of these materials are out of the scope of this paper and can be found in [24] to [26].

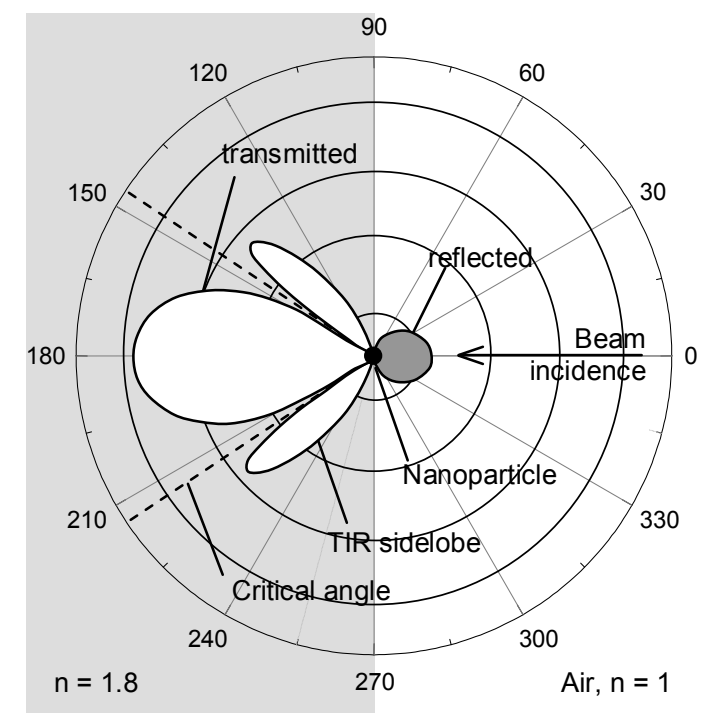

Fig. 4. Angular distribution of radiation scattered from an AZO nanoparticle located at an interface between air (refractive index $n=1)$ and a substrate with $n=1.8$

Based on the measured and calculated optical and plasmonic properties of these nanoparticles, we performed numerical simulation of the IR radiation scattering on them. In accordance with the experimental data quoted in [24] to [26] we considered a spherical dipole TCO nanoparticle with a radius $35 \mathrm{~nm}$, located at an interface between a semi-infinite medium with a refractive index value $n=1$ and a layer with $n=1.8$. In our simulation the beam was assumed to arrive perpendicularly to the detector surface at a wavelength of $1.4 \mu \mathrm{m}$ (near infrared). For our calculation we utilized Eqs. (3) to (8) and applied the approach to scattering cross section determination as outlined in [27]. Fig. 4 shows a large enhancement of scattering cross section, together with preferential distribution of the incident power into the photodetector in the direction of the incident beam and with an appearance of sidelobes in the simulated distribution due to the total internal reflection (TIR) effect. Thus, plasmonic nanoparticles made from alternative redshifted materials can be used for the enhancement of photodetector operation in the infrared wavelength range. The presented case corresponds to the situation shown in Fig. 3a, and the active region is located on the left from the nanoparticle. It should be emphasized here that the radial distribution shown in Fig. 4 is related with a single plasmonic nanoparticle only (its dimensions and properties being defined above) and does not directly reflect the overall photodetector element performance which is further defined by the specific detectivity enhancement.

A further redshifting strategy for plasmonic nanoparticles is to embed them into a medium with a value of refractive index higher than that of the nanoparticle [28]. The influence of the embedding medium with a higher value of dielectric permittivity was numerically assessed utilizing a simplified scattering model from [29]. The dielectric permittivity of the nanoparticle was determined using the simple mixing model, $\left(\varepsilon_{\text {total }}=\left[\varepsilon_{\text {particle }}+\varepsilon_{\text {medium }}\right] / 2\right)$.

The normalized cross-section is defined here in the usual manner as the ratio of the power scattered by a particle versus the total power intercepted by it. The simulated results shown in Fig. 5 show that an increase of the dielectric permittivity of the surrounding medium shifts the cross-section maximum towards longer infrared wavelengths. A very similar effect had previously been reported for metal plasmonic nanoparticles at shorter wavelengths [28].

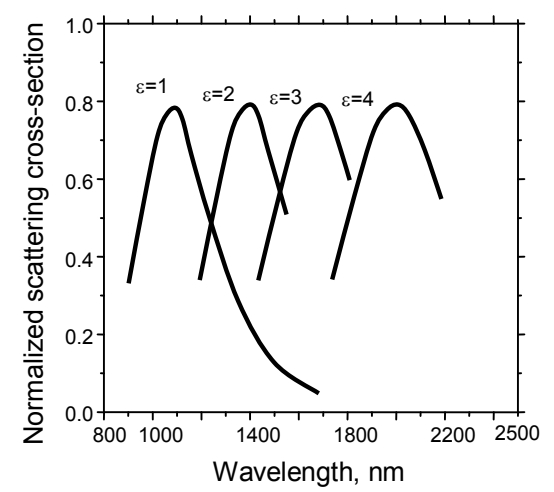

Fig. 5. Assessment of the influence of embedding medium permittivity (shown in figure for each corresponding curve) to the scattering cross-section of TCO nanoparticles; for easier comparison, all peaks are normalized to the same value

Another method to tailor the position of the scattering cross-section peak is to influence the density of nanoparticles, i.e. to change the distance between them, as proposed in [30], where the authors considered silica nanospheres with gold shell, i.e. hollow plasmonic nanoparticles. The influence of the distance between separate nanoparticles to the 
redshifting of the normalized scattering cross-section peak was assessed, as shown in Fig. 6. It can be seen that a decrease of the distance between nanoparticles causes a shift of the peak toward longer wavelengths. A detailed account on the redshifting due to the interaction of closely adjacent nanoparticles based on finite element modeling can be found in [31].

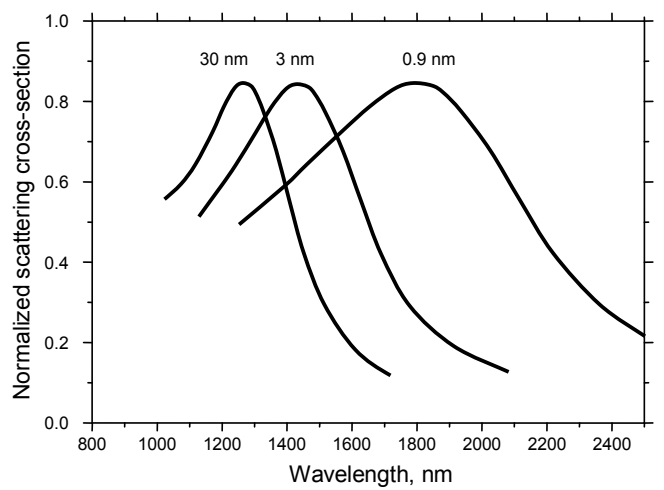

Fig. 6. Assessment of the influence of interparticle distance (shown in figure for each corresponding curve) to the scattering cross-section of TCO nanoparticles; for easier comparison, all peaks are normalized to the same value

An important point is how to physically implement the proposed plasmonic enhancement to the existing types of IR HgCdTe detectors. This question reduces to the technology of surface functionalization utilizing thin layers of dispersed nanoparticles [32]. One of the convenient methods to deposit nanoparticles to the existing detector surface is drop coating, where a dispersion of nanoparticles in an inorganic liquid also containing some kind of surfactant agent is utilized. Our as-produced experimental TCO nanoparticles are already dispersed in benzyl alcohol [24], thus facilitating the procedure. The deposition itself is done using a capillary syringe ensuring accurate control of the deposited amount. After the deposition, the mixture is left to settle, allowing nanoparticles to self-assemble on the photodetector surface. TCO may be deposited in the described manner directly onto the active surface or onto the surface passivation/ antireflection coating. An important point here is that in the latter case the dielectric or heterostructural passivation layer obviously must be much thinner than the localized evanescent field depth.

Regarding the achievable improvements of the photodetector figures of merit, it should be born in mind that light trapping through plasmonic enhancement effectively increases the photodetector optical thickness $\alpha d$ (where $\alpha$ is the absorption coefficient and $d$ the physical thickness of the photodetector). Due to this reason, the best performance enhancement is expected for very thin detector structures. According to [9], plasmonic light trapping ensures 10- to 100-fold shrinkage of the active region thickness while keeping $\alpha d$ constant. In the first experiments with plasmonic enhancement of photovoltaic photodetectors an approximately 20 -fold increase of the photocurrent was observed. The assessment of realistic uncooled $\mathrm{HgCdTe}$ photoconductors gives a specific detectivity improvement of about 2 to 2.5 , which is strongly dependent on the actual thickness of the lowest $\mathrm{Cd}$ molar fraction part of the epitaxial layer.

An advantage of our proposed strategy to utilize alternative plasmonic material like TCO and apply the described redshifting approaches is that transparent conductive oxides inherently have lower losses than the standard plasmonic materials like gold, silver, etc. Thus, we are able to place our nanoparticles at the front surface of the detector (the geometry shown in Fig. 2c) and at the same time to avoid excessive parasitic absorption losses. This is in contrast to metal nanoparticles to be placed near the back side of the detector (which is at the same time technologically more demanding). Also, according to [9], in the case of nanoparticles at the front surface, light will first acquire an angular spread within the active region and thus enhance absorption to subsequently reflect from the detector back side, returning again to the front nanoparticles and thus ensuring re-radiation via the identical scattering mechanism, consequently furnishing an even higher efficiency. Thus, the use of TCO may give us an additional degree of freedom when designing photodetectors and permits us to avoid unnecessary technological changes in the production cycle of photodetector chips, which contributes to simplicity and cost effectiveness.

\section{CONCLUSION}

We analyzed the possibility to enhance the specific detectivity of narrow-bandgap IR detectors utilizing plasmonic nanoparticles. To this purpose, we considered redshifting the absorptance maximum of the nanoparticles utilizing alternative plasmonic materials belonging to the class of transparent conductive oxides, the immersion of such nanoparticles into higher refractive index medium and the adjustment and tailoring of the packaging density of nanoparticles. In all cases the nanoparticles are stochastically distributed directly on the photodetector surface. An advantage is that TCO plasmonic nanoparticles may simultaneously serve as scatterers to enhance the capture of the incident radiation 
and as subwavelength optical "antennas" directly increasing absorption in the photodetector active area through field concentration caused by the presence of evanescent plasmon modes. The redshifting of the signal can help achieve operating wavelengths reaching the mid-infrared range. A further patterning and the application of designer plasmon using regular subwavelength patterns may ensure shifting to the far-infrared part of the spectrum. In this manner, thin film-based uncooled IR detectors could become a viable alternative for different applications, even the high-end ones like the use in homing devices for smart ammunition.

\section{ACKNOWLEDGMENT}

The research presented in this paper was funded by the Ministry of Education and Science of the Republic of Serbia within the projects TR 32008 and III 47029.

\section{REFERENCES}

[1] Rogalski, A. (2010). Infrared Detectors. CRC Press, Bocca Raton.

[2] Gupta, S.K., Saxena, S., Singhal, A., Ghosh, A.K. (2008). Trajectory correction flight control system using pulse jet on artillery rocket. Defence Science Journal, vol. 58, no. 1, p. 15-33.

[3] Vogt, R., Glebocki, R. (2000). Impulse Control of Anti-Tank Mortar Missile. RTO AVT Symposium on Active Control Technology for Enhanced Performance Operational Capabilities of Military Aircraft, Land Vehicles and Sea Vehicles, Braunschweig.

[4] Gunapala, S.D., Rhiger, D.R., Jagadish, C. (2011). Advances in Infrared Photodetectors. Academic Press, Amsterdam.

[5] Djurić, Z., Piotrowski, J., Jakšić, Z., Djinović, Z. (1988). Ambient temperature HgCdTe photoconductor can achieve detectivity higher than $1 \cdot 10^{8} \mathrm{~cm} \mathrm{~Hz}^{1 / 2} / \mathrm{W}$ at $10.6 \mu \mathrm{m}$. Electronics Letters, vol. 24, no. 25, p. 15901591.

[6] Djinović, Z., Djurić, Z., Jakšić, Z., Kermendi, F., Roknić, R. (1991). Isothermal vapor phase epitaxy of (Hg,Cd) Te from Te-rich Hg1-yTey source. Journal of Crystal Growth, vol. 108, no. 3-4, p. 710-718, DOI:10.1016/0022-0248(91)90251-Y.

[7] Jović, V., Djurić, Z., Jakšić, Z., Popović, M. (1994). Composition profiles of $(\mathrm{Hg}, \mathrm{Cd}) \mathrm{Te}$ liquid phase epitaxy layers grown from Te-rich solution. Journal of Crystal Growth, vol. 143, no. 3-4, p. 176-183, DOI:10.1016/0022-0248(94)90053-1.

[8] Jakšić, Z., Djurić, Z. (2004). Cavity enhancement of auger-suppressed detectors: A way to backgroundlimited room-temperature operation in 3-14 $\mu \mathrm{m}$ range. IEEE Journal on Selected Topics in Quantum Electronics, vol 10, no. 4, p. 771-776.
[9] Atwater, H.A., Polman, A. (2010). Plasmonics for improved photovoltaic devices. Nature Materials, vol. 9 , no. 3, p. 205-213, DOI:10.1038/nmat2629.

[10] Ferry, V.E., Munday, J.N., Atwater, H.A. (2010). Design considerations for plasmonic photovoltaics. Advanced Materials, vol. 22, no. 43, p. 4794-4808, DOI:10.1002/ adma.201000488.

[11] Ferry, V.E., Verschuuren, M.A., Li, H.B.T., Verhagen, E., Walters, R.J., Schropp, R.E.I., Atwater, H.A., Polman, A. (2010). Light trapping in ultrathin plasmonic solar cells. Optics Express, vol. 18, no. 13, p. A237-A245, DOI:10.1364/OE.18.00A237.

[12] Mokkapati, S., Beck, F.J., De Waele, R., Polman, A., Catchpole, K.R. (2011). Resonant nano-antennas for light trapping in plasmonic solar cells. Journal of Physics D: Applied Physics, vol. 44, no. 18, p. 185101, DOI:10.1088/0022-3727/44/18/185101.

[13] Schuller, J.A., Barnard, E.S., Cai, W., Jun, Y.C., White, J.S., Brongersma, M.L. (2010). Plasmonics for extreme light concentration and manipulation. Nature Materials, vol. 9, no. 3, p. 193-204, DOI:10.1038/nmat2630.

[14] Maier, S.A. (2007). Plasmonics: Fundamentals and Applications. Springer Science+Business Media, New York.

[15] Chang, C.C., Sharma, Y.D., Kim, Y.S., Bur, J.A., Shenoi, R.V., Krishna, S., Huang, D., Lin, S.Y. (2010). A surface plasmon enhanced infrared photodetector based on InAs quantum dots. Nano Letters, vol. 10, no. 5, p. 1704-1709, DOI:10.1021/nl100081j.

[16] Rosenberg, J., Shenoi, R.V., Vandervelde, T.E., Krishna, S., Painter, O. (2009). A multispectral and polarization-selective surface-plasmon resonant midinfrared detector. Applied Physics Letters, vol. 95, no. 16, p. 161101, DOI:10.1063/1.3244204.

[17] Pendry, J.B., Martín-Moreno, L., Garcia-Vidal, F.J. (2004). Mimicking surface plasmons with structured surfaces. Science, vol. 305, no. 5685, p. 847-848, DOI:10.1126/science.1098999.

[18] Boltasseva, A., Atwater, H.A. (2011). Low-Loss Plasmonic Metamaterials. Science, vol. 331, no. 6015, p. 290-291, DOI:10.1126/science. 1198258.

[19] Drude, P. (2005). The Theory of Optics. Dover Publications, Mineola, New York.

[20] Lorentz, H.A. (1880). Über die Beziehungzwischen der Fortpflanzungsgeschwindigkeit des Lichtes der Körperdichte. Annalen der Physik, vol. 9, p. 641-665.

[21] Lorentz, H.A. (1952). The theory of electrons, Dover Publications, Mineola, New York.

[22] Bohren, C.F. Huffman, D.R. (1983). Absorption and scattering of light by small particles. John Wiley \& Sons, New York.

[23] Jackson, J.D. (1999). Classical electrodynamics. John Wiley \& Sons, New York.

[24] Niederberger, M., Garnweitner, G., Buha, J., Polleux, J., Ba, J., Pinna, N. (2006). Nonaqueous synthesis of metal oxide nanoparticles:Review and indium oxide as case study for the dependence of particle morphology on precursors and solvents. Journal of Sol-Gel Science 
and Technology, vol. 40, p. 259-266, DOI:10.1007/ s10971-006-6668-8.

[25] Bilecka, I., Niederberger, M. (2010). Microwave chemistry for inorganic nanomaterials synthesis. Nanoscale, vol. 2, p. 1358-1374, DOI:10.1039/ b9nr00377k.

[26] Jakšić, Z., Vuković, S., Buha, J., Matović, J. (2011). Nanomembrane-Based Plasmonics. Journal of Nanophotonics, vol. 5, p. 051818-1-21.

[27] Schmid, M., Klenk, R., Lux-Steiner, M.C., Topič, M., Krč, J. (2010). Modeling plasmonic scattering combined with thin-film optics. Nanotechnology, vol. 22, no. 2, p. 025204, DOI:10.1088/09574484/22/2/025204.

[28] Beck, F.J., Polman, A., Catchpole, K.R. (2009). Tunable light trapping for solar cells using localized surface plasmons. Journal of Applied Physics, vol. 105, 114310-1-7, DOI:10.1063/1.3140609.

[29] Le Ru, E., Etchegoin, P. (2009). Principles of surfaceenhanced raman spectroscopy and related plasmonic effects, Elsevier, Amsterdam.

[30] Ung, T., Liz-Marzán, L.M., Mulvaney, P. (2001). Optical properties of thin films of $\mathrm{Au} @ \mathrm{SiO}_{2}$ particles. Journal of Physical Chemistry B, vol. 105, no. 17, p. 3441-3452, DOI:10.1021/jp003500n.

[31] Khoury, C.G., Norton, S.J., Vo-Dinh, T. (2009). Plasmonics of 3-D nanoshell dimers using multipole expansion and finite element method. ACS Nano, vol. 3, p. 2776-2788, DOI:10.1021/nn900664j.

[32] Jakšić, Z., Matovic, J. (2010). Functionalization of Artificial Freestanding Composite Nanomembranes. Materials, vol. 3, p. 165-200, DOI:10.3390/ ma3010165. 